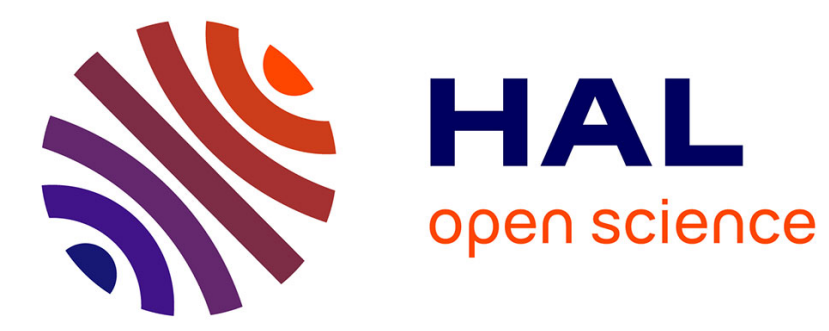

\title{
Graph constrained-CTM observer design for the Grenoble south ring
}

Carlos Canudas de Wit, Luis Ramon Leon Ojeda, Alain Kibangou

\section{To cite this version:}

Carlos Canudas de Wit, Luis Ramon Leon Ojeda, Alain Kibangou. Graph constrained-CTM observer design for the Grenoble south ring. CTS 2012 - 13th IFAC Symposium on Control in Transportation Systems, Sep 2012, Sofia, Bulgaria. hal-00728555

\section{HAL Id: hal-00728555 \\ https://hal.inria.fr/hal-00728555}

Submitted on 6 Sep 2012

HAL is a multi-disciplinary open access archive for the deposit and dissemination of scientific research documents, whether they are published or not. The documents may come from teaching and research institutions in France or abroad, or from public or private research centers.
L'archive ouverte pluridisciplinaire HAL, est destinée au dépôt et à la diffusion de documents scientifiques de niveau recherche, publiés ou non, émanant des établissements d'enseignement et de recherche français ou étrangers, des laboratoires publics ou privés. 


\title{
Graph constrained-CTM observer design for the Grenoble south ring
}

\author{
Carlos Canudas-de-Wit ${ }^{*}$ Luis Leon Ojeda** \\ Alain Y. Kibangou ${ }^{* * *}$ \\ * GIPSA-Lab, CNRS, Dept. of Control Systems, NeCS team, Grenoble, \\ France. (e-mail: carlos.canudas-de-wit@gipsa-lab.grenoble-inp.fr). \\ ** INRIA, NeCS Team, Grenoble, France (e-mail: luis.leon@inria.fr) \\ *** GIPSA-Lab,CNRS, University Joseph Fourier, NeCS team, \\ Grenoble, France (e-mail: alain.kibangou@ujf-grenoble.fr)
}

\begin{abstract}
An important problem in traffic estimation, forecasting, and control is the reconstruction of densities in portions of the road links not equipped with sensors. In this paper, and based on ideas from Morarescu and Canudas-de Wit [2011], we use a deterministic constrained model that reduces the number of possible affine dynamics of the system and preserves the number of vehicles in the network. In particular we reformulate the idea in Morarescu and Canudas-de Wit [2011] with the correct number of feasible modes, and introduce the concept of graph constrained-CTM observer, which is used to reconstruct the densities from the Grenoble south ring use case that contains 45 cells organized in 9 links, and is simulated using a calibrated AISUM micro-simulator. This work is performed in connection with HYCON2 traffic show case (www.hycon2.eu), and with the Grenoble Traffic Lab (GTL) (http://necs.inrialpes.fr/pages/reseach/gtl.php).
\end{abstract}

Keywords: Highway traffic estimation; virtual sensors; traffic observers.

\section{INTRODUCTION}

Reconstructing densities in portions of the road links not equipped with sensors constitutes an important task in traffic estimation, forecasting, and control problems. Among many other approaches, model-based observers is one popular technique to build this information. They can also be understood as "virtual sensors" deployed inside of the cells not equipped with "true sensors". They are used to better track, in real-time, density variations with a fine degree of granularity in the space, as the "virtual cells" can be selected as small as desired.

The efficient real-time traffic conditions estimation using only a limited amount of data is a challenging problem since the traffic is described by a system which is observable only when a segment situated between two vehicle detector stations (sensors) is entirely congested or entirely free, Muñoz et al. [2003], Muralidharan and Horowitz [2009].

Macroscopic models such as the Cell Transmission Model (CTM) are often used as basis to build observers. After a first tuning, when these models are validated ( Lin and Ahanotu [1994]) they can be used for several purposes. One possibility is to design open-loop state-estimation (Jia et al. [2001]) where the open-loop model is reproduced without any corrective term. One of the main difficulty when dealing with the density estimation for the traffic network is due to the hybrid dynamics (several operation modes) governing the behavior of the system (Gomes et al. [2008], Kurzhanskiy [2009], Muñoz et al. [2003]). Some works have used random switches between modes to cope with uncertainties in this switching process. In addition, this requires the estimation of the probability density function associated with these variations. Reported results show that the density can be fairly well estimated. However, the observation does no longer respect the conservation law (vehicles may appear/disappear in the network), Kurzhanskiy [2009].

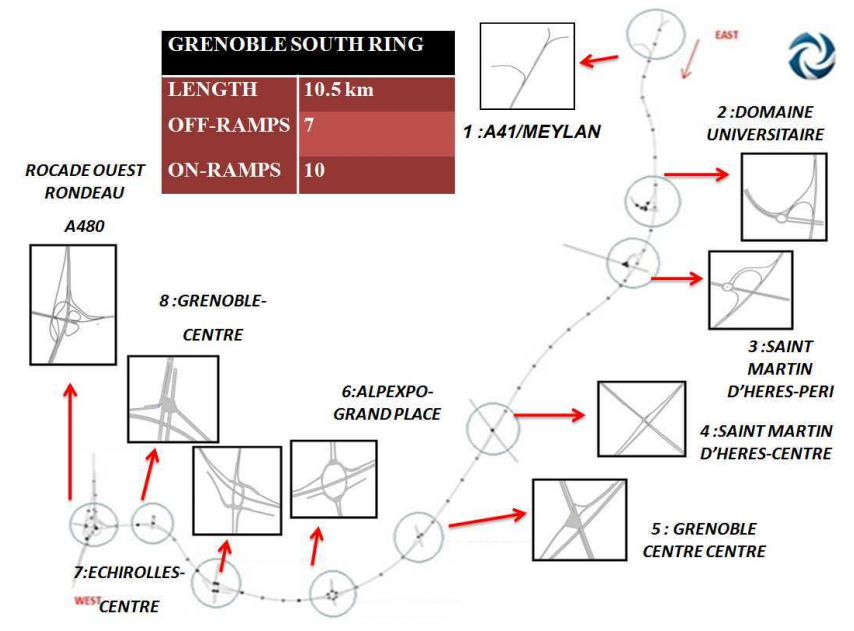

Fig. 1. Grenoble South Ring

In this paper, we use a deterministic constrained CTM model that reduces the number of possible affine dynamics of the system and preserves the number of vehicles in the network. This idea was originally proposed in the paper Morarescu and Canudas-de Wit [2011], but the proposed constrained model resulted in an oversimplified number 
of modes. In this paper we reformulate this idea with the correct number of feasible modes, and introduce the concept of graph constrained-CTM observer, which is used to reconstruct the densities from the Grenoble south ring use case that contains 45 cells. The test are done using a calibrated AISUM micro-simulator including 9 links of a road sector of $10.5 \mathrm{Km}$. The paper presents also a detailed evaluation of the observed performance along the full ring.

Note that other related problems such as imputation techniques to determine the missing on-ramp and off-ramp flows, Muralidharan and Horowitz [2009], and estimation of the origin-destination matrix, are not pertinent for our study case of the Grenoble south ring, as all the input and output ramps are equipped with sensors.

\section{PROBLEM FORMULATION}

The paper focuses on the study of ring-road network designed as a sequence of nodes relied by links, having relative small distances between ramps, i.e. the length of a link is smaller than $4 \mathrm{~km}$, (see Fig. 1 for the case of the Grenoble South Ring). We consider setups where sensors are located at the node level with our main concerning being the estimation of the density inside the links, each link being partitioned in several cells. In that, it is worth to notice that each observation problem is decentralized at the link level; the density of the cells belonging to a link is estimated using only the data provided by the sensors located at the link boundaries.

\subsection{Macroscopic CTM-model}

The traffic dynamics models are based on the car conservation principle. One of the most popular macroscopic traffic flow model used for the analysis of highway traffic behavior is the cell transmission model (CTM) proposed by Daganzo [1994] which is based on the LWR model introduced in Lighthill and Whitham [1955] and Richards [1956]. The CTM is a macroscopic traffic model particularly well designed for model-based control and estimation, owing to its analytical simplicity and ability to reproduce congestion wave behavior. Due to the particular set-up of the Grenoble south ring, we can consider all segments with one measurable entry and one exit (without on-ramp and/or off-ramp), partitioned in $N$ cells.

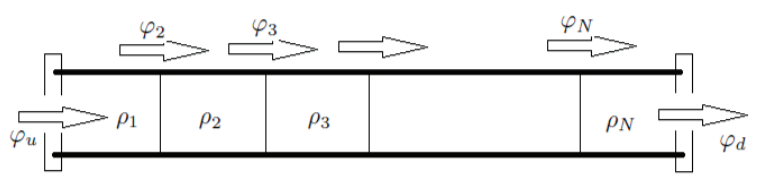

Fig. 2. The cell transmission model.

The CTM model can be written as:

$$
\rho_{i}(k+1)=\rho_{i}(k)+\frac{T}{l_{i}}\left(\varphi_{i}(k)-\varphi_{i+1}(k)\right)
$$

where $\rho_{i}(k)$, is the density of the $i$ th cell in a link at a specific time indexed by $k$, and $\varphi_{i}(k)$ is the flow at the cell interface. If we denote $T$ the discrete time interval, $l_{i}$ the cell length, and $v_{i}$ the free-flow speed, it is well known that the condition $v_{i} T<l_{i}$ is sufficient for guaranteeing convergence of (1) LeVeque [1992].
The CTM is a piecewise linear state-dependent model in which the flow is computed as the minimun of the supply $S_{i}$, and the demand $D_{i-1}$ at each interface

$$
\varphi_{i}=\min \left\{D_{i-1}, S_{i}\right\}
$$

with

$$
\begin{aligned}
D_{i-1} & =\min \left\{v_{i-1} \rho_{i-1}, \varphi_{m, i-1}\right\}, \\
S_{i} & =\min \left\{\varphi_{m, i}, w_{i}\left(\rho_{m, i}-\rho_{i}\right)\right\}
\end{aligned}
$$

where $\varphi_{m, i}$ is the maximum flow allowed by the capacity of cell $i, \rho_{m, i}$ is the jam density (i.e. the maximum density that can be reached), $v_{i}$ corresponds to the free flow speed and $w_{i}$ is the congestion wave speed in cell $i$. All these parameters can be considered as different, but for simplicity sake we assume that they are the same within a link. Nevertheless, they are allowed to be different at different links. They will be identified from the microsimulator as it will be explained in latter sections.

In the sequel and with the purpose to light the notation, we will refer to a single link with parameters $\left(v, w, \rho_{m}, \varphi_{m}, \rho_{c}\right)$, where $\rho_{c}$ is the critical density where the flow is maximal.

\subsection{Graph constrained CTM-model}

Model (1) has different operation modes. Indeed, according to $(2)$ each flow interface $\varphi_{i}$ can be defined either by its upstream demand $D_{i-1}$, or by its downstream supply $S_{i}$ depending what is the minimum. In that, each interface flow has two possible modes and therefore each cell has 4 possible operation modes. Nevertheless, as the output flow of upstream cells is the input flow for the downstream one, and as we have a total of $N+1$ flow interfaces in a $N$-cell link, the maximum number of modes is

$$
M=2^{(N+1)} \text {. }
$$

If we assume that only one congestion wave may exist in a highway link, and that it will propagate upstream, then the number of modes can be reduced, and a constrained model can be devised. This idea was originally proposed in Morarescu and Canudas-de Wit [2011]. However the proposed constrained model resulted in an oversimplified number of modes. In this sub-section we restate this idea with the correct formulation. To this aim, the following definitions are in order.

Definition 1. A cell $i, i=1, \cdots, N$, is considered free $(\mathrm{F})$ if its associated density $\rho_{i}$ is equal or below its critical density $\rho_{c}$, else it is considered to be congested $(\mathrm{C})$ if $\rho_{c}<\rho_{i} \leq \rho_{m}$.

Definition 2. A cell interface flow $\varphi_{i}, i \in\{1, \ldots, N+1\}$ is said to be advective, if $\varphi_{i}=S_{i}$, and it is said to be diffusive, if $\varphi_{i}=D_{i-1}$. In other words, advective (respectively, diffusive) flows describes waves traveling upwards (respectively, downwards) through the interface. The interface flow will be denoted as $\overleftarrow{\varphi}_{i}$ and $\vec{\varphi}_{i}$ for the advective and diffusive case respectively.

Note that $\varphi_{1}$ and $\varphi_{N+1}$ denote respectively the upstream flow $\varphi_{u}$ and the downstream flow $\varphi_{d}$.

Now, using the above definitions and the assumption that congestions always appear at cell $N$ and propagate upstream, the restricted number of modes can be evaluated. Indeed, under such a hypothesis only the following cell 
combinations do exist: $F F, F C, C C$ (the case of $C F$ is ruled out by the working hypothesis). For each of these 3 combination we have:

- $F_{i-1} F_{i}: \varphi_{i}=\vec{\varphi}_{i}=D_{i-1}$ is diffusive

- $C_{i-1} C_{i}: \varphi_{i}=\overleftarrow{\varphi}_{i}=S_{i}$ is advective

- $F_{i-1} C_{i}: \varphi_{i}$ is diffusive if $\left(D_{i-1} \leq S_{i}\right)$ or advective if $\left(D_{i-1}>S_{i}\right)$.

Now taking a 3-cells example, we have the following possible combinations: $F F F, F F C, F C C$, and $C C C$. Noticing that each interface of the type $F C$ can have two possible modes, then, including the upstream and downstream interface flows, we can identify a total of 8 different modes, that we can classify as shown in Table 1 , where $\overrightarrow{\mid}$ and $\overleftarrow{\mid}$ indicate when the flow interface is diffusive or advective, respectively. The modes are indicated alternatively by the integer variable $s(k)$ or by the associate letter with the upper arrow indicating the direction of the waves. Using the letter notation will be useful to easily distinguish diffusive modes, $\overrightarrow{(\cdot)}$ to the advective ones $\overleftarrow{(\cdot)}$

Table 1. Operating mode table

\begin{tabular}{|c|c|c|}
\hline Mode $s(k)$ & Mode $\stackrel{\leftrightarrows}{\leftrightarrows}$ & Cells configuration \\
\hline \hline 1 & $\vec{a}$ & $F F F \overrightarrow{\mid}$ \\
2 & $\overleftarrow{a}$ & $F F F \overleftarrow{\mid}$ \\
3 & $\vec{b}$ & $F F \overrightarrow{\mid} C$ \\
4 & $\overleftarrow{b}$ & $F F \overleftarrow{\mid} C$ \\
5 & $\vec{c}$ & $F \overrightarrow{\mid} C C$ \\
6 & $\overleftarrow{c}$ & $F \overleftarrow{\mid} C C$ \\
7 & $\vec{d}$ & $\overrightarrow{\mid} C C C$ \\
8 & $\overleftarrow{d}$ & $\overleftarrow{\mid} C C C$ \\
\hline
\end{tabular}

The total number of modes in the constrained case, is then reduced to

$$
M=2(N+1) .
$$

These modes have an associated graph $\mathcal{G}$ as the transition between modes are not arbitrary, but they follow the next rules:

- only one congestion wave may exist in the considered link, and that it will propagate upstream following the pattern: $F F F, F F C, F C C, C C C$,

- Transitions $F F \rightarrow F C$ are only possible from an advective to a diffusive mode,

- Transitions $F C \rightarrow F F$ are only possible from a diffusive to a diffusive mode.

The last two properties can be derived from continuity of the fundamental diagram during the transient phases. Details of the graph are shown in Fig. 3. The figure also illustrated the possible graph paths when the congestion moves upwards and downwards.

Now, let us give the state-space representation of the system where the vector $\rho=\left(\rho_{1}, \ldots, \rho_{N}\right)$ denotes the state of the system, the measured data used by the system being the upstream and downstream flows $\left(\varphi_{u}, \varphi_{d}\right)$ and the upstream and downstream velocities $\left(v_{u}, v_{d}\right)$. The index $s(k) \in\{1,2, \ldots, M\}$ is used in order to precise the mode of the entire highway segment.

With this notation the system dynamics are given by:

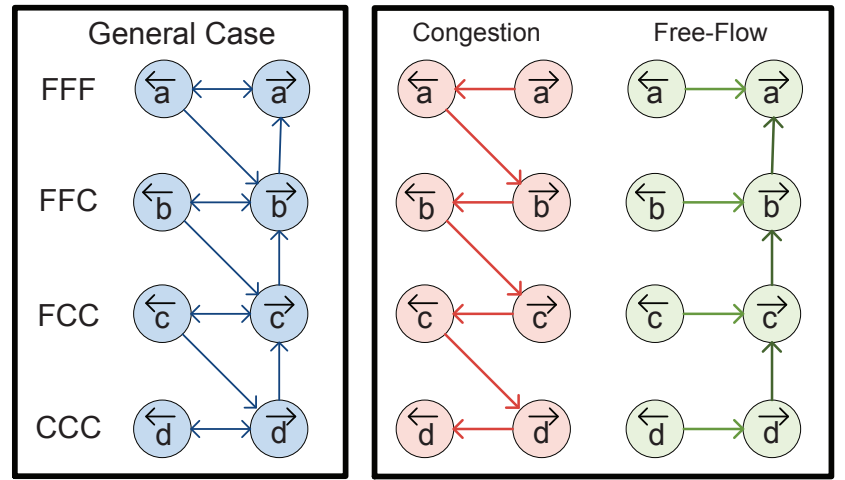

Fig. 3. Illustration of the Graph $\mathcal{G}$ associated to the constrainedCTM model, for an example of 3-Cells. The left Figure show the general case with all possible modes. The center figure show the graph path for when congestion moves upwards, and the right figure show the graph path when the congestion moves downwards.

$$
\left\{\begin{array}{l}
\rho(k+1)=A_{s(k)} \rho(k)+B \varphi(k)+\bar{B}_{s(k)} \rho_{m} \\
s(k)=\mathcal{G}(\rho(k), \varphi(k)) \\
y(k)=h(\rho(k), s(k))
\end{array}\right.
$$

where $\varphi=\left(\varphi_{u}, \varphi_{d}\right)$ is the input,

$$
h(\rho(k), s(k))=\left\{\begin{array}{l}
v \rho_{N}(k) \text { if } s(k)=1 \\
w\left(\rho_{m}-\rho_{1}(k)\right), \text { if } s(k)=M \\
0, \text { otherwise }
\end{array}\right.
$$

Exact definition of the matrices $A_{s(k)} \in \mathbb{R}^{N \times N}, B \in$ $\mathbb{R}^{N \times 2}, \bar{B}_{s(k)} \in \mathbb{R}^{N \times 1}, \forall i \in\{1,2, \ldots, M\}$ can be found in Morarescu and Canudas-de Wit [2011], the main difference being the total number of modes $M$, and the associated Graph $\mathcal{G}$.

Remark 1. Fundamental differences between the "standard" CTM model (1)-(2), and the constrained-CTM model (3) is that under small perturbations due to measurement noise or model uncertainties, the constrainedCTM model (3) preserves its graph structure, whereas the CTM model (1)-(2) could produce infeasible changes of modes. In that, the constrained-CTM model offers additional physical-consistency properties coherent with the Gudonov's solutions and the vehicle preservation law.

\section{OBSERVER DESIGN}

\subsection{Observability}

The observability of the CTM model for different SMM modes has been studied elsewhere, see for example works in Muñoz et al. [2003] and the references therein. The results are summarized in Table 2 .

Table 2. Observability for different SMM modes

\begin{tabular}{|c|c|c|}
\hline Up. Cells & Down. Cells & Observable with \\
\hline \hline Free-flow & Free-flow & Downstream measurement \\
\hline Congested & Congested & Upstream measurement \\
\hline Congested & Free-flow & Up. and Down. measurement \\
\hline Free-flow & Congested & Unobservable \\
\hline
\end{tabular}

In mode $s(k)=1$, i.e. the $F \ldots \vec{F}$ case, $\varphi_{u}$ is not related to the density of the cell and it is seen as an external input 
to the system, whereas $\varphi_{d}=v \rho$ is state dependent, and hence can be associated to the output of the system, i.e.

$$
y=\varphi_{d}=v \rho_{N}=C_{1}^{T} \rho, \quad C_{1}^{T}=[0,0, \ldots, 0, v]
$$

the system is then backward observable (i.e. using downstream measurements).

In mode $s(k)=M$, i.e. the $\overleftarrow{C} \ldots C$ case, $\varphi_{d}$ is measured and $\varphi_{u}=w\left(\rho_{m}-\rho\right)$. Thus we have a reversed situation in which $\varphi_{d}$ is seen as an input and $\varphi_{u}$ is state dependent, and can be associated to the output of the system, i.e.

$$
y=w\left(\rho_{m}-\rho\right)=C_{M}^{T} \rho+w \rho_{m}, \quad C_{M}^{T}=[-w, 0, \ldots, 0,0]
$$

In this case the system is forward observable (i.e. using upstream measurements).

Except for combinations of the type $C F$ (ruled out here), all other modes are non observable because neither $\varphi_{u}$, nor $\varphi_{d}$ depend on the density of the cell. The system is then unobservable but the system is open-loop stable, which allows for internal state predictions (without feedback).

\subsection{Observer structure}

A hybrid observer that uses only the segment boundary conditions can be built from the basis of equation (3) by reproducing these dynamics and activating a correcting term when the modes are observable

$$
\left\{\begin{aligned}
\hat{\rho}(k+1)= & A_{\hat{s}(k)} \hat{\rho}(k)+B \varphi(k)+\bar{B}_{\hat{s}(k)} \rho_{m}+ \\
& +K_{\hat{s}(k)}(y(k)-h(\hat{\rho}(k), \hat{s}(k)) \\
\hat{s}(k) \quad & \mathcal{G}(\hat{\rho}(k), \varphi(k))
\end{aligned}\right.
$$

where $K_{\hat{s}(k)}$ is the observer gain. This gain depends on the estimated modes and is used only for the observable modes $\hat{s}(k)=1$ and $\hat{s}(k)=M$. The design is as follows:

- spectral radius of $\left(A_{1}-K_{1} C_{1}\right)$ strictly lower than 1 for $\hat{s}=1$,

- spectral radius of $\left(A_{M}-K_{M} C_{M}\right)$ strictly lower for $\hat{s}=M$,

- $K_{\hat{s}}=0$ for $\hat{s}=2,3, \ldots, M-1$.

To improve the performance with respect to the open-loop estimator, by using an adequate pole placement method, the gains $K_{1}$ and $K_{M}$ are selected such that the spectral radius of $\left(A_{1}-K_{1} C_{1}\right)$ (resp. $\left.\left(A_{M}-K_{M} C_{M}\right)\right)$ be upperbounded by the lowest eigenvalue of $\mathbf{A}_{1}\left(\operatorname{resp} . \mathbf{A}_{M}\right)$.

\section{DESCRIPTION OF THE USE CASE}

Grenoble South Ring ("Rocade sud" in French) is approximately $10.5 \mathrm{~km}$ long and is made up by two carriageways of two-lanes motorways each. For our study case only the direction east-west was modeled. In this section of the highway there are 10 on-ramps and 7 off-ramps. It is considered as a use case within the framework of the FP7 NoE HYCON2 (Highly-Complex and Networked Control Systems), and its main goal is to provide a rich set of field traffic data to the control community in order to test control technologies of networked hybrid systems in largescale real-world problems.

The first step for preparing the study case was the development of a microscopic model for Grenoble south ring using the traffic simulator Aimsun. Once achieved the correct architecture of the highway, we proceeded with the identification of the macroscopic characteristics, such as split ratio and demand flows, necessary to tune the whole microscopic simulator. To define the flow going through the whole network the demand flows in the 13 entrances were provided. These demand profiles were obtained by collection of real data in a typical traffic scenario spanning a period of 24 hours from 12:00 am to 12:00 am. On the other hand, knowing that the split ratio is defined as the percentage of flow from the demand that goes through the off-ramp. The methodology adopted was a top to bottom procedure, starting from the node A41/Meylan to the last node Rondeau. This procedure was based on the modification of the split ratio for each node, starting at Domaine Universitaire and carrying onto Grenoble-Center (see figure 1) and our final objective was to obtain the same demand profile at the last node (Rondeau). This tuning was made for a 5 minutes time step and a 24 hours time horizon.

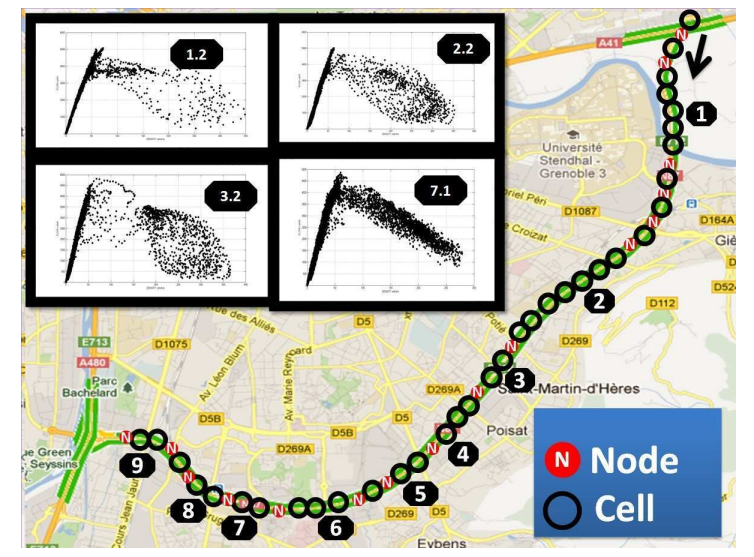

Fig. 4. Grenoble South Ring Cell partition.

Using cell densities instead of cell occupancies permits the CTM to include uneven cells, which leads to greater flexibility in partitioning the highway. Moreover smaller cells allow better numeric accuracy. For the observer application on the entire highway, Grenoble south ring was divided into 9 links (see Fig. 4 and Table 3).

Table 3. Grenoble south ring cell partition

\begin{tabular}{|l|l|l|l|l|l|l|l|l|l|}
\hline Link\# & 1 & 2 & 3 & 4 & 5 & 6 & 7 & 8 & 9 \\
\hline \#cells & 5 & 7 & 2 & 3 & 2 & 3 & 2 & 2 & 2 \\
\hline
\end{tabular}

For data collection, virtual sensors on Aimsun were located at the boundaries of each link, as well as of each cell(validation purposes). The length of all cells were chosen to be as homogeneous as possible, for instance, for link 1 the 5 cells were as big as $220 \mathrm{~m}$, while for the link 2 the 7 cells were $226 \mathrm{~m}$. As stated in previous sections, for numerical stability reasons, the time and space cell size should verify the condition $v_{i} T_{s} \leq l_{i}, i=1 \ldots N$. Therefore, the simulation time-step should be lower than 9 seconds; we used $8 \mathrm{sec}$. Note also that data was filtered using a 1storder Butterworth lowpass filter with cutoff frequency 10 $\mathrm{mHz}$.

The characteristic curve parameters were estimated with an off-line identification process using first-order model 
and a least square approximation method. The table 4 shows all cell parameters for the first link (see Fig.4).

Table 4. Fundamental diagram parameters for link 1

\begin{tabular}{ccccc} 
Link & $\mathrm{v}$ & $\mathrm{w}$ & $\rho_{c}$ & $\rho_{m}$ \\
\hline First cell & 70 & 19 & 90 & 410 \\
Second cell & 72 & 15 & 60 & 396.66 \\
\hline
\end{tabular}

\section{SIMULATION RESULTS}

In this section, we present the simulation results obtained for the entire highway. We first introduce a Space-Time figure that illustrates congestion behavior on Grenoble south ring for the simulated densities and the reconstructed ones. As a second point, the estimated and measured densities in a 24 hours simulation will be given for a cell, we then present a comparison between the open and closed-loop observers using the cumulative distribution function, and last the Instanteneous Travel Time as a performance metric is provided.

Fig.5 (top) depicts the behavior of congestion for a typical day on Grenoble south ring. It is clear that one of the most important points of congestion is at the last node (Le Rondeau). This point represents a real congestion problem for the travelers in Grenoble. It is also visible that the strong traffic jams correspond with the morning and afternoon time period (8:00 am and 17:00 pm) when people either go or come back from work. As expected this congestion propagate backwards. The Fig.5 (bottom) shows how the observer is able to estimate the density profiles on the highway.
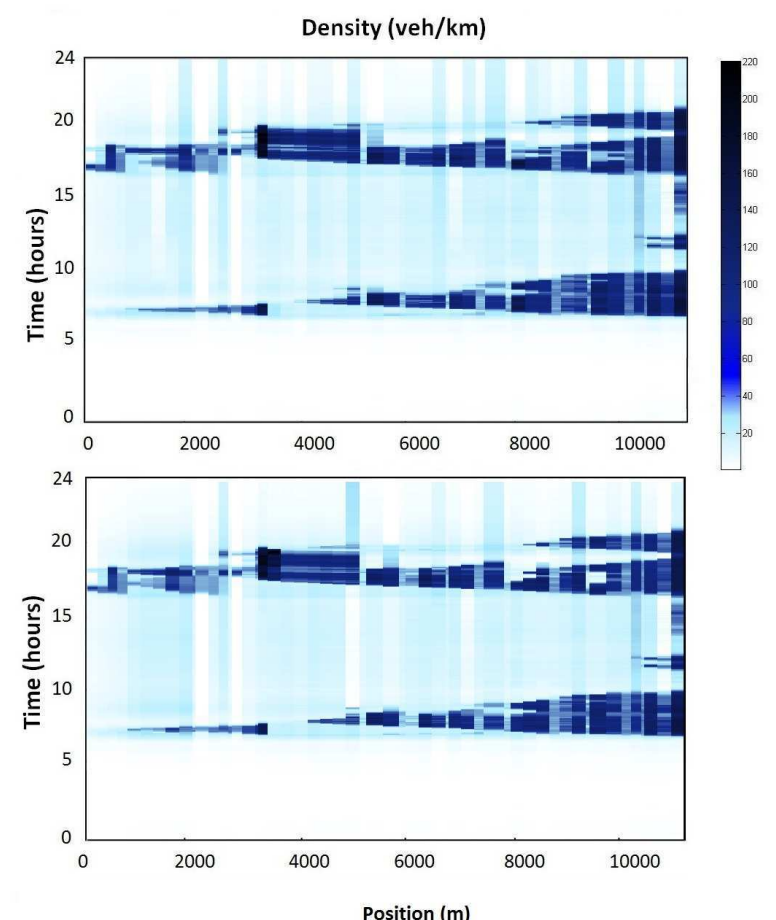

A/41 Meylan Rondeau

Fig. 5. Space Vs Time traffic condition (Measured: Top, Estimated: Bottom)

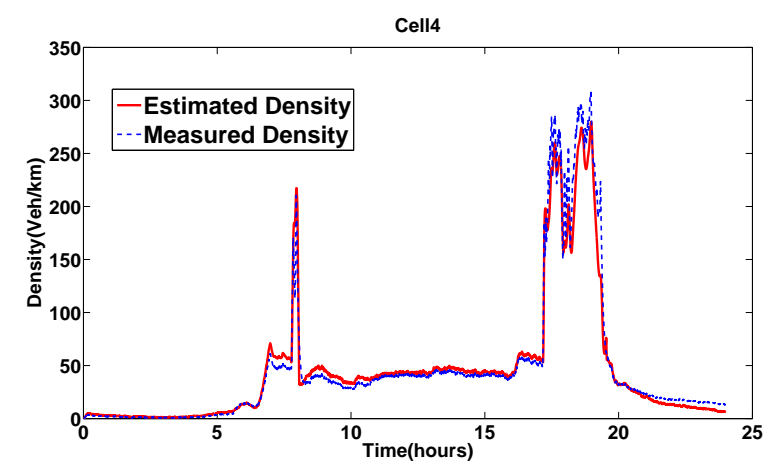

Fig. 6. Measured and estimated densities for cell 4 of link 2.

In order to show the observer results on a specific segment of the highway, the link 2 was chosen. This section is $1.58 \mathrm{~km}$ long and it was divided into 7 cells of 227 $\mathrm{m}$ approximately each (see Fig.4). Only the 4th cell is presented. This figure exhibits more in detail the two important periods during the day. During the morning around 8:00 am, a not very strong congestion propagates backwards. In contrast, in the afternoon around 17:00 pm a strong congestion can be seen. It is clear that the developed observer performed well: the estimated traffic density was close to the simulated traffic density.

Now, we evaluate the performance of the observer by using the root mean square (RMS) error as performance index. For a link with $n$ cells, the RMS is defined as

$$
R M S(k)=\sqrt{\frac{1}{n}\|\rho(k)-\hat{(k)}\|^{2}}
$$

where $\rho(k)$ and $\rho \hat{(k)}$ denote respectively the actual and the estimated densities. Using the measured errors for all the links and for the 24 hours time-window, we have evaluated the empirical cumulative distribution function, which is depicted in Fig.7 for the open loop and closed loop observers. As it can be seen, applying closed loop increases the probability to be less than or equal to certain performance index. More precisely, we can note that $90 \%$ of time the RMS is lower than $34 \mathrm{veh} / \mathrm{km}$ that corresponds to approximately $10 \%$ of error. Such performance is obtained with the open-loop observer only $80 \%$ of time.

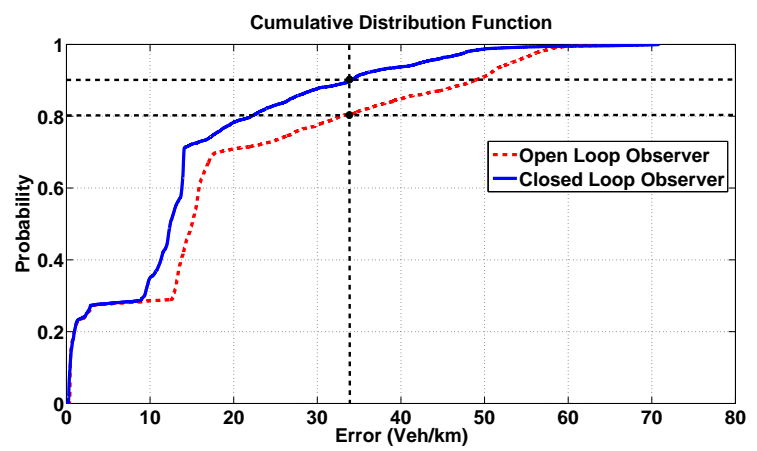

Fig. 7. Cumulative Distribution Function for the open loop and closed loop observer during the 24 hours simulation for the global highway

Now we evaluate the performance in terms of the Instantaneous Travel Time. This metric permits to develop 
strategies that improve traffic performance on congested road networks:

$$
\begin{gathered}
I T T(t)=\sum_{l=1}^{N} \operatorname{ITT}_{l}(t), \text { with } \\
\operatorname{ITT}_{l}(t)=\frac{\Delta x_{l}}{V_{l}(t)}
\end{gathered}
$$

where $V_{l}(t)$ is the traffic speed in kilometers per hour $(\mathrm{km} / \mathrm{h})$, $\rho_{l}(t)$ is the density in vehicle per kilometers (veh/ km), $\Delta x_{l}$ is the link length in kilometers $(\mathrm{km})$, and $l$ is the link considered.

The Fig. 5 depictes the performance. The starting and ending point from which the ITT was computed was the start and the end of Grenoble south ring respectively. Hence the figure shows at each time step how long it would take for a driver to go across the entire highway. It can be easily seen how a fairly good estimation is obtained. The Table 5 shows the standard deviation, mean error, and the normalized RMS error for the density and Instantaneous Travel Time estimation; it is worth noting that these values are obtained through the comparison between the measured and estimated values of density and ITT. It is clear that the observer feedback adds a corrective term that improves the response of the system. The normalized error, after introducing the feedback, decreases a $34 \%$ for the density estimation, while it decreases a $13.14 \%$ for the instantaneous travel time estimation. On the other hand, the mean RMS error for the open-loop density estimation is located at $18.59 \mathrm{veh} / \mathrm{km}$ while for the closed-loop is at $14.19 \mathrm{veh} / \mathrm{km}$, obtaining a reduction of $23.67 \%$ and $23.39 \%$ for its standard deviation. Whereas the mean RMS error and standard deviation for ITT estimation decreases a $33.62 \%$ and $71 \%$ respectively from open-loop to closedloop. The normalized RMS error is computed as:

$$
N R M S=\frac{R M S}{\|\rho(k)\|} \times 100 \%
$$

where $\rho(k)$ and $\rho(\hat{k})$ are defined as above.

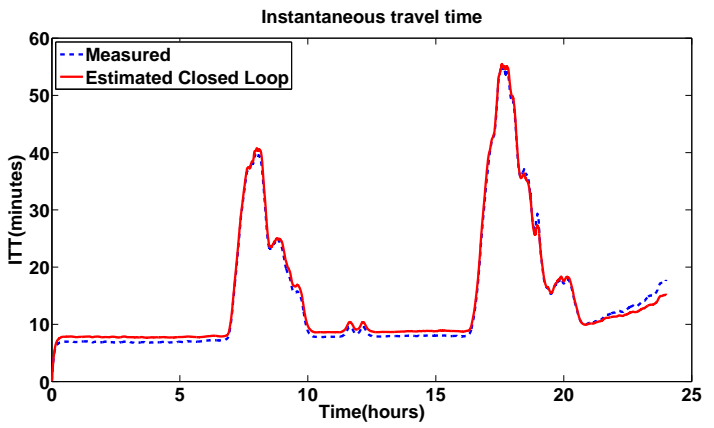

Fig. 8. Instantaneous Travel Time (ITT).

\section{CONCLUSIONS}

In this paper, the estimation for traffic densities using a deterministic constrained model based on CTM model was investigated. We have shown that with the introduction of
Table 5. Performances error

\begin{tabular}{|c|c|c|c|c|} 
& \multicolumn{2}{c}{ Density (Veh/km) } & \multicolumn{2}{c}{ ITT (minutes) } \\
\cline { 2 - 5 } NMRS(\% ) & O. Loop & C. Loop & O. Loop & C. Loop \\
\cline { 2 - 5 } Mean & 14.09 & 9.30 & 8.07 & 7.01 \\
\cline { 2 - 5 } Std & 18.59 & 14.19 & 1.16 & 0.77 \\
\cline { 2 - 5 } & 16.72 & 12.81 & 1.17 & 0.34 \\
\cline { 2 - 5 } & & & &
\end{tabular}

a graph constrained-CTM observer we are able to reconstruct rather accurately the internal states (densities) of a road portion not equipped with sensors. This strategy for real-time density estimation was applied on Grenoble South Ring. Simulation results exhibit that the measured densities obtained from the traffic simulator Aimsun and the estimated densities agree closely. We are currently working to improve the procedure for the system parameters identification, as well as a more optimal approach to compute the observer gain. Additionally, we are investigating the presented technique to provide a suitable prediction of performance measures, such as, traffic speed, travel-time and vehicle hours travelled, among others.

\section{ACKNOWLEDGEMENT}

The research leading to these results has received funding from the European Union Seventh Framework Programme [FP7/2007-2013] under grant agreement no $257462 \mathrm{HY}-$ CON2 Network of excellence, and the MOCoPo.

\section{REFERENCES}

C.F. Daganzo. The cell transmission model: A dynamic representation of highway traffic consistent with the hydrodynamic theory. Transportation Research Board, 28(4):269-287, 1994.

G. Gomes, R. Horowitz, A.A. Kurzhanskiy, J. Kwon, and P. Varaiya. Behavior of the cell transmission model and effectiveness of ramp metering. Transportation Research, C, 16(4):485-513, 2008.

Z. Jia, C. Chen, B. Coifman, and P. Varaiya. The pems algorithms for accurate, real-time estimates of g-factors and speeds from single-loop detectors. In Proc. of IEEE Intelligent Transportation Systems Conference, pages 536-541, 2001.

A.A. Kurzhanskiy. Set-valued estimation of freeway traffic density. In Proc. of 12th IFAC Symposium on Control in Transportation Systems, 2009.

R.J. LeVeque. Numerical methods for conservation laws. Birkhäuser, 1992.

M.J. Lighthill and G.B. Whitham. On kinematic waves ii: A theory of traffic flow on long crowded roads. Proceedings of the Royal Society of London. Series A, Mathematical and Physical Sciences, 229(1178):317-345, 1955.

W.-H. Lin and D. Ahanotu. Validating the basic cell transmission model on a single freeway link. Path technical note 95-3, Institute of Transportation Studies, University of California at Berkeley, 1994.

I.-C. Morarescu and C. Canudas-de Wit. Highway traffic modelbased density estimation. In Proc. of IEEE American Control Conference, San Francisco, CA, USA, June 2011.

L. Muñoz, X. Sun, R. Horowitz, and L. Alvarez. Traffic density estimation with the cell transmission model. In Proc. of IEEE American Control Conference, pages 3750-3755, 2003.

A. Muralidharan and R. Horowitz. Imputation of ramp flow data for freeway traffic simulation. Transportation Research Record, pages 58-64, 2009.

P.I. Richards. Shock waves on the highway. Operations Research, 4 (1):42-51, 1956. 